\title{
An Evaluation of the Antioxidants of Phytochemical Origin as a Source of Antihyperglycemic Agents in Controlling Diabetes in
} Albino Rats

\author{
Saramma G* \\ Department of Biomedical Sciences, University of Botswana, Botswana
}

*Corresponding author: George Saramma, Department of Biomedical Sciences, Faculty of Medicine, University of Botswana, Gaborone, Botswana, Email: saramma.george@mopipi.ub.bw

\section{Mini Review}

Volume 1 Issue 2

Received Date: May 17, 2018

Published Date: May 30, 2018

\section{Abstract}

The present study was aimed at the in-vitro and in-vivo analysis of the antioxidant status of three different plants which are commonly used in traditional medicine in Botswana and to evaluate its antidiabetic activities. The plants used in this study were the aerial parts of Ocimum gratissimum, Momordica balsamina and corms of Hypoxis hemerocallidea. It was further investigated that whether their antihyperglycemic effects are working through the antioxidant system. All the results obtained in different experiments indicate that the antioxidant status of these extracts was positive and the antidiabetic activity is working through this antioxidant system.

Keywords: Antihyperglycemic Effect; Antidiabetic Potential; Antioxidant Activity; $\alpha$-Amylase; Reactive Oxygen Species (ROS); Phytochemicals

Abbreviations: ROS: Reactive Oxygen Species; DM: Diabetes Mellitus; WHO: World Health Organization; LDLs: Low Density Lipo-Proteins; HIV: Human Immuno deficiency Virus; MEMB: Methanol Extract of $M$ Balsamina; MEOG: Methanol Extract of O.gratissimum; MEHHC: Methanol Extract of Hypoxis Hemerocallidea Corm; TBA: Thiobarbituric Acid; TPC: Total Phenolic Content; OGTT: Oral Glucose Tolerance Test; ALT: Alanine amino Transferase; AST: Alanine Aspartate Transaminase; ALP: Alkaline Phosphatase.

\section{Introduction}

Phytochemicals are naturally occurring and biologically active plant compounds that have potential capabilities of inhibiting oxidative stress induced by many external factors. Plants synthesize complex materials which have no immediate obvious growth or metabolic functions which are referred as secondary metabolites and one of such secondary metabolites is the phenols. Common categories of phenols are flavonoids, tannins and lignin's and the flavonoids have remarkable antioxidant properties that can prevent the formation of damaging free radicals. Free radicals can cause cellular damages through metabolic activation of those compounds to highly reactive substances such as reactive oxygen species (ROS). Recent years, numerous traditional medicinal plants were tested for their antidiabetic potential in the experimental animals [1]. Complementary and alternative medicine involves the use of herbs and other dietary 


\section{International Journal of Zoology and Animal Biology}

supplements as alternatives to mainstream allopathic medical treatment.

Diabetes mellitus (DM) is becoming one of the leading causes of death worldwide, is a metabolic disorder characterized by chronic hyperglycemia with altered secretion or inefficient utilization of insulin. The impaired metabolism is often accompanied with excessive release of free radicals through lipid peroxidation, due to alteration of the activity of several proteins, aggravated by a drastic drop in antioxidant immune mechanisms. This imbalance generally lead to oxidative stress which is the main factor associated with the severity and death in diabetes [2].

The World Health Organization (WHO) has listed 21,000 plants which are used for medicinal purposes around the world. Among them, 150 species are used commercially on a fairly large scale [3]. Even though many of studies have been conducted in this area, could not reveal the actual mode of action of the above plants. So the major aim of this study was, therefore, to examine the possible hypoglycaemic effects of the methanolic extracts of the above plants, with a view of providing a pharmacological rationale for this extracts in the management of adult-onset, Type- 2 diabetes mellitus.

The studies were conducted in three different steps as follows:

a) Plants

b) in- vitro studies

c) In-vivo studies

\section{Plants}

Hypoxis hemerocallidea belongs to the star lily family (Hypoxidaceae). It is commonly known as the African potato. It is a tuberous plant with linear or broad lanceshaped leaves which are hairy. The leaves can measure about $400 \mathrm{~mm}$. The flowers usually close at midday and are star-shaped with a bright yellow colour. This plant has recently attracted a lot of research interest because of its possible immune stimulant properties in its tuberous part. The healing properties may be a result of phytochemicals which are antioxidants and phytosterols [4] which may reduce the low density lipo-proteins (LDLs) in the blood. Traditionally it has been used to treat many conditions like hypertension, diabetes mellitus and arthritis.

Momordica balsamina, also known as the African pumpkin, is a short-lived wild climber which belongs to
Cucurbitaceae family. It has bright orange fruits with small spikes on the surface. M. balsamina has high protein content as well as a variety of important micronutrients like potassium, phosphorus, calcium, magnesium, iron and zinc [5]. Its leaves, fruits, seeds and bark offer a wide range of medicinal use including immune stimulant properties. The healing properties of the plant are due to chemicals such as resins, alkaloids, flavonoids, glycosides, steroids and terpenes [5]. The leaves and young fruits can be eaten after cooking, but the ripe fruit can cause diarrhea and vomiting. Traditionally it is used as an analgesic and to treat conditions like excessive uterine bleeding, cardiovascular disorders, gastrointestinal problems, diabetes mellitus, syphilis and rheumatism.

Ocimum gratissimum is a shrub belonging to Lamiaceae family. It is commonly known as the African basil or wild basil. It can reach heights of 1-2 meters, with ribbed stem and simple leaves which are oppositely arranged. It may have antimicrobial properties because it is traditionally used to treat conditions like diarrhea, respiratory infections, and eye and skin disorders [3]. This plant is also commonly used in Ayurvedic medicine [6]. Recent studies on Ocimum gratissimum proved to be a useful medication for people living with Human Immuno deficiency Virus (HIV), and Acquired Immuno Deficiency Syndrome AIDs [7].

All the plants were collected locally from Botswana, and all of them are seasonal. The voucher specimens were submitted in the herbarium of the University of Botswana. For the African potato the corms were cut into small pieces, dried in the shade, coarsely powered and for O.gratissimum and $\mathrm{M}$. balsamina the aerial parts are used and extracts were prepared in $70 \%$ methanol. The extracts obtained were used to carry out the experiments as MEOG (Methanol extract of O.gratissimum), MEMB (Methanol extract of $M$. balsamina) and MEHHC (Methanol extract of Hypoxis hemerocallidea corm) respectively. All the chemicals used were of analytical grade and bought from Sigma-Aldrich Chemical Company, (St. Louis, MO) USA. The kits for bioassays were bought from Agape Switzerland. The solvents used for the extraction process were also of analytical grade. Male albino rats of Wistar strain (Rattusnorvegicus) of body weight ranging 200-250 grams were housed in colony cages at ambient temperatures of $25^{\circ} \mathrm{C} \pm 2^{\circ} \mathrm{C}$ and $50-55 \%$ relative humidity with 12 Hours light and dark cycle. They had water and food ad libitum. Experiment was conducted as per the internationally accepted principles for laboratory animal care unit of University of Botswana. 


\section{International Journal of Zoology and Animal Biology}

\section{In- vitro Studies}

This includes the phytochemical screening to identify the phytochemicals present and the study revealed that all of these extracts showed the presence of phenols, flavonoids and tannins. The antioxidant status were checked with different assays such as DPPH assays both TLC and Spectrophotometric methods described by Yeboah, et al. [8]. Total antioxidant status was estimated by ABTS radical scavenging activity and was determined by the method described by Pellegrini, et al. [9]. Thiobarbituric Acid (TBA) assay was conducted to estimate the TBA present and the method followed was described by Rezaeizadeh, et al. (2011) [10]. The total phenolic content (TPC) of the extracts was determined using the Folin-Ciocalteu reagent method as described by Yeboah, et al. [8]. All the plants studies here showed a notable antioxidant activity in different systems including TBA, DPPH and ABTS assays.

Antidiabetic activity was evaluated by assessing the inhibitory potential of the plant extracts against $\alpha$ amylase using spectrophotometric assays. A gradual increase in the activity was shown in all the extracts together with acarbose which was used as a standard in a dose dependent manner [11].

\section{In- vivo studies}

In- vivo studies were conducted in rats as acute oral toxicity test, oral glucose tolerance (OGTT) in normal rats, acute glucose test in normal rats and acute/single dose glucose response test in STZ-induced diabetic rats and all the results were giving a positive effect on antihyperglycemic activity.

\section{In-Vivo Analysis}

Rats were divided into 8 groups of six each in each group, diabetes was induced by STZ one normal control, one diabetic control, and 3 groups of positive controls after diabetic induction treated with known antidiabetic drugs which were used for comparison with the extract treated groups. 3 groups of experimental groups after diabetic induction treated with 3 different extracts with the dose of $300 \mathrm{mg} / \mathrm{Kg}$ body weight. Treatment was carried out for 30 days and at the end of the experimental period final fasting blood glucose levels and body weights were recorded. Rats were anaesthetized and blood samples were collected from the aorta. Then the blood was centrifuged at $3500 \mathrm{rpm}$ for 15 minutes. Once centrifuged, the plasma was transferred into cryotubes, frozen in liquid nitrogen and stored at -80 degrees Celsius for biochemical estimations.

\section{Biochemical Analysis}

TBARS was estimated by the method described by Niehaus, et al. [12]. Glucose levels were determined by the use of the glucometer. Hb1Ac, total proteins and total albumen were estimated by kits from Agape Switzerland and their instructions were strictly followed. Alanine aminotransferase (ALT), alanine aspartate transaminase (AST) alkaline phosphatase (ALP) were estimated by the kits bought from Sigma and the manufacture's guidelines were followed. The lipid profile was done with kits bought from Agape Switzerland and their instructions were strictly followed. This includes the triglyceride, LDL, HDL and total cholesterol [13].

The overall results indicated that all the parameters checked in the above experiments showed a positive response in antihyperglycemic effect. The liver function indices also showed a significant decrease in experimental groups which were treated with the extracts when compared with the diabetic control. The results of the lipid profile also showed a significant reduction in LDL and total cholesterol [14]. Based on the findings of this study the methanol extracts of the above plants showed anti -hyperglycemic effect in different assays checked. The active source of the antioxidants present in these extracts indicated that this activity is mainly through its antioxidant system. Further studies need to be carried out to isolate and investigate the individual active principles. Some medicinal plants are known to possess both hyper- and hypoglycaemic constituents. Therefore, isolation of the individual compounds would optimize the therapeutic effects of the plant.

\section{References}

1. Dinkova Kostova AT (2008) Phytochemicals as Protectors against Ultraviolet Radiation: Versatility of Effects and Mechanisms. Planta Med 749(13): 15481559.

2. Dailey G (2011) Overall mortality in diabetes mellitus: where do we stand today?. Diabetes Technol Ther 13(1): 65-74.

3. World Health Organization (2009) Legal Status of Traditional Medicines and Complementary/Alternative Medicine: A Worldwide Review, WHO Publishing. 


\section{International Journal of Zoology and Animal Biology}

4. Boukes GJ, Van de VM (2016) In vitro modulation of the innate immune response and phagocytosis by three Hypoxis spp. and their phytosterols. South African J Botany 102: 120-126.

5. Thakur GS, Bag M, Sanodiya BS, Bhadouriya P, Debnath M, et al. (2009) Momordica balsamina: A medicinal and neutraceutical plant for health care management. Curr Pharma Biotechnol 10(7): 667682.

6. Joshi RK (2013) Chemical composition, in vitro antimicrobial and antioxidant activities of the essential oils of Ocimum gratissimum, 0 . sanctum and their major constituents. Indian J Pharm Sci 75(4): 457-462.

7. Elujoba AA (2000) Studies on the anti diarrhoea activity of Ocimum gratissimum. University of Ile Ife Press.

8. Yeboah EM, Majinda RR (2009) Radical scavenging activity and total phenolic content of extracts of the root bark of Osyris lanceolata. Nat Prod Commun 4(1): 89-94.

9. Re R, Pellegrini N, Proteggente A, Pannala A, Yang M, et al. (1999) Antioxidant activity applying an improved ABTS radical cation decolorization assay. Free Radic Biol Med 26(9): 1231-1237.

10. Rezaeizadeh A, Zuki ABZ, Abdollahi M, Goh YM, Noordin MM, et al. (2011) Determination of antioxidant activity in methanolic and chloroformic extracts of Momordica charantia. Afri J Biotechnol 10(24): 4932-4940.

11. Hansawasdi C, Kawabata J, Takanori K (2000) $\alpha$ amylase inhibitors from Roselle (Hibiscus sabdariffa Linn.) tea. Biosci Biotechnol Biochem 64(5): 10411043.

12. Niehaus WG, Samuelson B (1986) Formation of MDA from phospholipid arachidonate during microsomal lipid peroxidation. Eur J Biochem 6(1): 126-130.

13. Arise RO, Aderounmu IG, Oguntibeju OO (2014) Lipid Profile, Antidiabetic and Antioxidant activity of Acacia ataxacantha Bark extract in Streptozotozin-induced Diabetic Rats. Intech Open Science.

14. Van Wyk BE (2011) The potential of South African plants in the development of new medicinal products. South African J Botany 77(4): 812-829. 